\title{
Comparison of Super Hydrophobic Mechanism of Several Typical Plant Leaves
}

\author{
Yanhong Sun ${ }^{1, a}$ and $\mathrm{Xu}$ Wang \\ ${ }^{1}$ Jilin Engineering Normal University, Changchun, China \\ a343175460@qq.com
}

Keywords: Plant leaf; Super hydrophobic; Contact angle

\begin{abstract}
Through the observation of the typical plant lotus leaf, rice and nasturtium etc leaf surface wettability, the leaf surface structure of scanning electron microscope, contact angle measurement, self cleaning mechanism of comparative study of typical plant leaf surface structure, clarifying the typical super hydrophobic plant surface wettability difference and its origin. The results showed that the leaf surface of the three kinds of plants had both micro - and nano - surface microstructure, and all of them showed super hydrophobicity. The microscopic morphology is composed of a micron scale mastoid substrate, which is covered with a nano scale waxy fluff, so that the water droplets can not be completely immersed into the substrate and exhibit the super hydrophobic property. The contact angle measurement results prove this point. But unlike the other two is that the super hydrophobic properties of rice leaves are anisotropic, rice leaf surface parallel to the edge of the mastoid leaf direction showed ordered one-dimensional, arbitrary in the vertical direction, water droplets in the rice leaf surface is easy to flow in parallel along the leaf edge direction. The study on the mechanism of wettability of the typical plant leaf surface provides an idea for the development of the surface structure and the preparation of superhydrophobic surface.
\end{abstract}

\section{Introduction}

Lotus (Nelumbo nucifera), also known as lotus. A perennial aquatic herb belonging to the family. On the lotus leaf, there is a long, hard and fluffy hair, and the lotus leaf itself is accompanied by biological wax, so the surface tension is very low. The bead is close to the perfect circle, rolling / sliding on the surface of the lotus leaf, and can not be attached to the surface of the lotus leaf. This phenomenon is called lotus effect (Lotus-Effect)". The current international research on lotus effect has made many important theoretical results ${ }^{[1-5]}$; in the application, "superhydrophobic" products have all kinds of imitation lotus market, such as oil and water repellent fabric, lotus effect latex paint, waterproof paint, hydrophobic film, self cleaning glass and so on ${ }^{[6-7]}$. The super hydrophobic and self-cleaning function of lotus leaf has aroused great interest of scientists and engineers. However, whether lotus leaf has the same or similar self-cleaning function under different conditions is still a topic that has not been studied deeply. For example, the surface of the lotus leaf surface in the fresh, old, sick, dry, such as the different state of the surface of the mastoid structure, chemical composition of the epidermis, and water contact angle, the angle of the similarities and differences and changes in the law rarely reported. The lotus leaf under different conditions on the form structure of scanning electron microscopy, and water contact angle measurement, lotus leaf wax extraction methods were observed and analyzed, in order to obtain the structure and composition of lotus leaf hydrophobic effect mechanism ${ }^{[8]}$.

\section{Test Equipment and Method}

Bionics(Bionics, Biomimetics, Biomimicry) is defined in many ways, and there is not yet a fully unified understanding. Steel, an American military surgeon, first proposed the concept of Bionics in $1960^{[1-4]}$. In 1998, the United States published a monograph on the use of bionics bionics Benyus ${ }^{[5]}$ new term (Biomimicry). Bionics is the imitation of biological science, which studies of biological systems, structure, material, function, energy conversion, information control features, and its application in technology system, to improve the existing technology and engineering equipment, the creation process, the new building automation. The number of leaves of fresh leaves, old leaves, diseased leaves and dead leaves of lotus leaf in Changchun city were collected as samples. Before the 
test, the leaves were washed with distilled water and then fixed by $7 \mathrm{~d}$ with the volume fraction of neutral buffer formalin fixative for. During the test, the leaves were dried and cut into $1 \mathrm{~cm}^{2}$ sample.

Using S-300N (Japanese manufacturing HITACHI) analysis of the morphology and structure of lotus leaf surface scanning electron microscope; application of DSA100 surface analysis system (German scholar Lu g Inc) sample measurement and surface water contact angle, water volume is $5 \mathrm{~L}$. The test specimen is placed in a test bench by the sessile drop method, liquid drops out of control hung on the tip, manual work table rise, make the specimen and the water drops from the contact tip, forming the base drops on the surface of the sample, the IMG1S-C type charge coupled device image sensor (CCD, charge coupled device (Shanghai) Angus Based Information Technology Co., Ltd. production) microscope color camera, online or offline determination of contact angle. Average value of each measurement 5 times. The forward and backward angles are measured at the 15 degree angle of the specimen.

The leaf of lotus leaf consists of cuticle and wax. Wax is made up of long chain high carbon fatty acid and long chain high carbon fatty alcohol and a small amount of free high carbon fatty acid, free high carbon fatty alcohol and high carbon alkane and so on. So in the test of chloroform (chloroform, $1.47 \sim 1.484 \mathrm{~g} / \mathrm{m}$ L Shanghai Ling Feng Chemical Reagent Co., Ltd.) as the extractant by solvent extraction method to extract the lotus leaf wax, influence of lotus leaf crude wax comparison under different conditions of hydrophobic. Extraction equipment has GH-252 type micro analytical balance (accuracy of $0.01 \mathrm{mg}$, the production of the Japanese company A \& amp;D), homemade extraction device, atmospheric distillation unit, etc.. The extraction steps: first, take fresh, old, withered disease, 4 typical state 10 lotus leaf g, into the constant pressure separating funnel; extraction device to chloroform and then add lotus leaf sample volume is about 10 times, extracting $1 \mathrm{~h}$ extraction at room temperature; after the completion of the filtered extract was distilled at atmospheric pressure distillation unit, concentrated to a certain volume after the removal of a small amount of residual solvents of water bath heating is obtained after the lotus leaf wax, with the quality of the electronic balance measurement of lotus leaf wax, with the relative percentage of crude wax quality and the quality of the samples as the extraction rate of extraction results. Average value of each measurement 5 times.Bionic information and its application

\section{Results and Analysis}

Observation and Measurement Results. Through observation, 4 typical states in fresh water, old, disease, dry lotus leaf surface wetting degree of figure 1a is a drop in fresh lotus leaf on the state of the water droplets together, forming a large drop of water, and can freely roll, showed a strong hydrophobic function; figure $1 \mathrm{~b}$ for lotus leaf in the aging state of water droplets in the distribution table on the leaves, although no longer fresh lotus leaf surface, leaf edge curl, water erosion, but the water is still clearly visible, hydrophobic properties still exist; figure 1C lotus leaf is dry yellow, leaf rust stains, belong to disease leaves, did not see the crystal clear water in the above "walk", hydrophobic properties disappear; figure 1D is the last stage of growth period of acquisition of the lotus leaf, the leaves have peeling, but without peeling, little drops of water or by twos and threes everywhere, the hydrophobic property is not inferior to the 2 . Therefore, the lotus leaf in the fresh, old, dry 3 kinds of state showed hydrophobic characteristics, and the disease leaf wettability with the other three different.

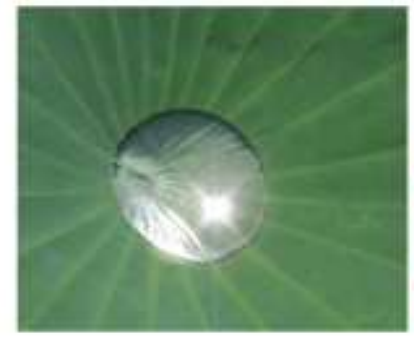

a.Fresh leaf

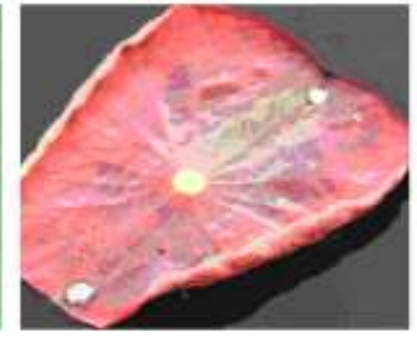

b.Old leaf

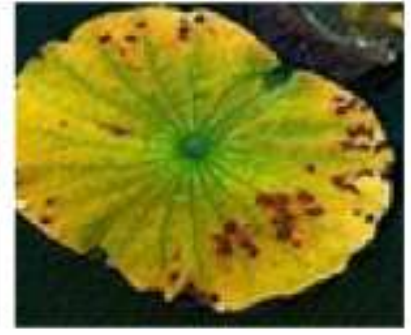

c.Sick leaf

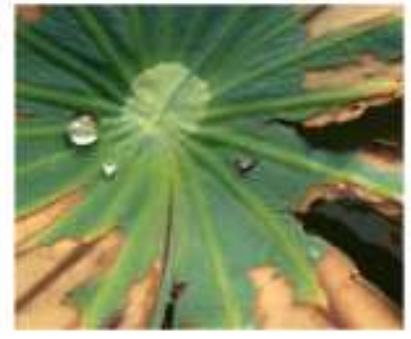

d.Dried-up leaf 


\section{Discussion and Analysis}

Fig.1 Wet states of four lotus samples

The sample is fixed on the stage, after spraying treatment, each group of 4 put together in a scanning electron microscope (SEM scanning, electron microscope) observed and photographed are shown in Figure 2 in the lotus leaf fresh, old, disease, dry under 4 conditions: SEM photo. Through the measurement of each sample scanning electron microscope observation and microscope found that fresh lotus leaf surface (Figure 2a) distribution of tiny "hemispherical" body of the mastoid, the diameter is about $10 \sim 15 \mathrm{~m}$, about $2 \sim 5 \mathrm{~m}$, mastoid spaced $10 \sim 20 \mathrm{~m}$, mastoid uniform size the density distribution, basically the same, about $3000 \sim 4000 / \mathrm{mm} 2$. In each micron mastoid body and wrapped with a layer of size is about $200 \sim 500 \mathrm{~nm}$ small waxy layer of hair (Figure $2 \mathrm{~b}$ ), the fresh leaves to form the two yuan micro nano composite microstructure; aging lotus leaf surface (Figure 2C) covered with fresh leaves than small conical papillae (body about $3 \sim 12 \mathrm{~m}$ ), the mastoid wax villi were compared with fresh leaves are short and sparse, uneven distribution of about $50 \sim 100 \mathrm{um}$, width of $5 \sim 20 \mathrm{~m}$ strip or cylindrical convex geometry, together constitute a rough surface structure of lotus leaf surface; the sick (Figure 2D) Lve Xianza random, mastoid, wax crystal two yuan composite structure of fresh lotus leaf disappeared, which covered the network structure body was almost completely mastoid ca. $10 \sim 30$ m linear fiber column, and fiber column without hair wax layer, the distribution of fiber column arranged in a crisscross pattern, no The law, no air layer formation conditions (Figure 2e); and the dried lotus leaf surface (Figure 2f) compared with the fresh leaf structure, micro nano composite microstructure of two yuan is still clearly visible, but the hemispherical body of fresh leaves into the original mastoid conical papillae, waxy leaves Mao Mingxian cashmere table smaller distribution not all, are not the same size.

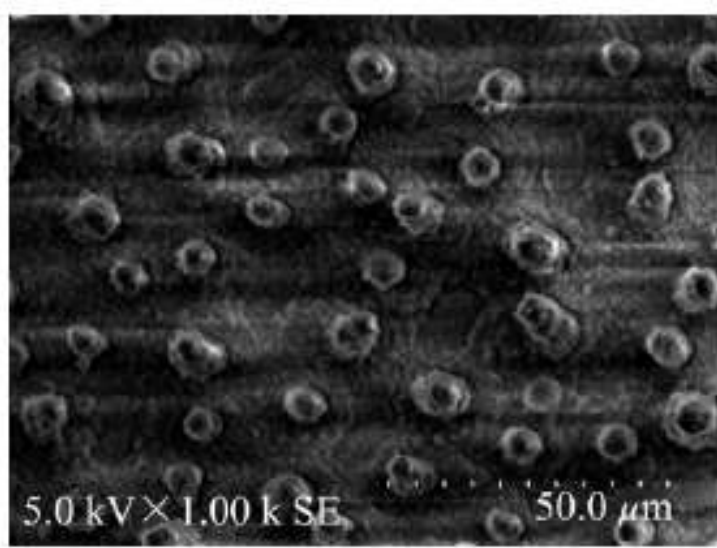

a. Fresh leaf $\times 1000$

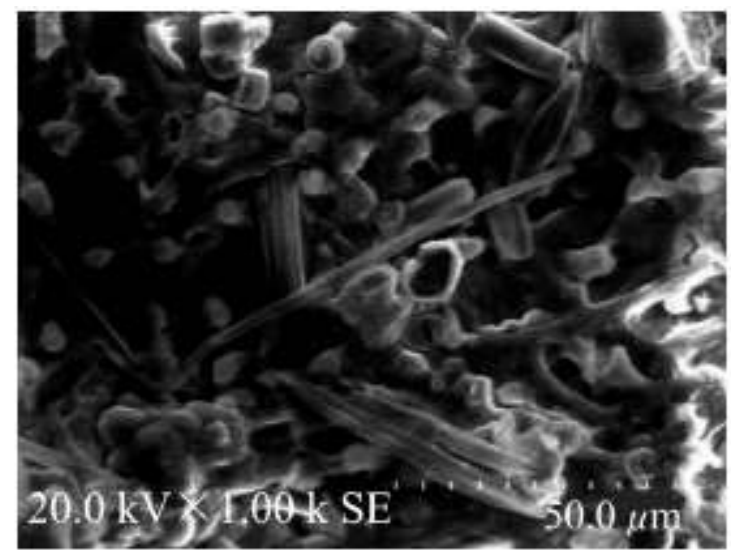

c. Old leaf $\times 1000$

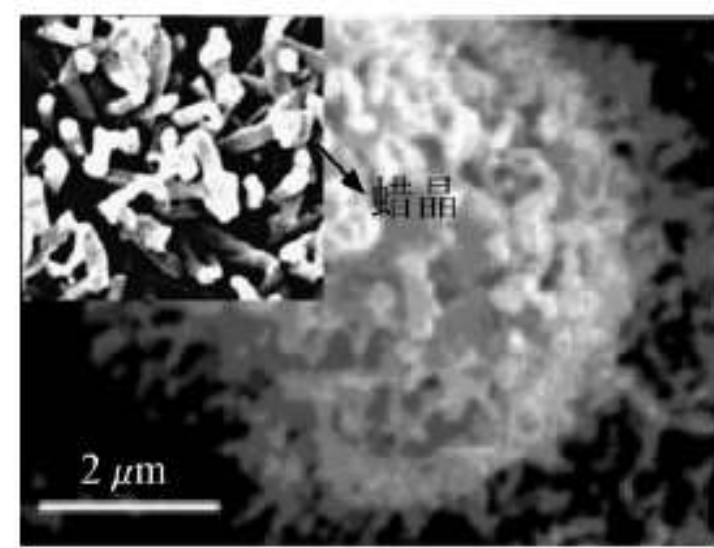

b. Fresh leaf' s single papillae

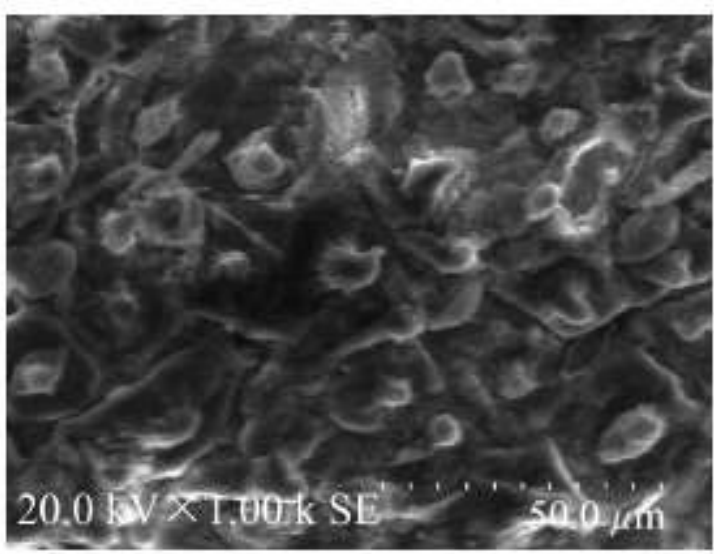

d. Sick leaf $\times 1000$ 


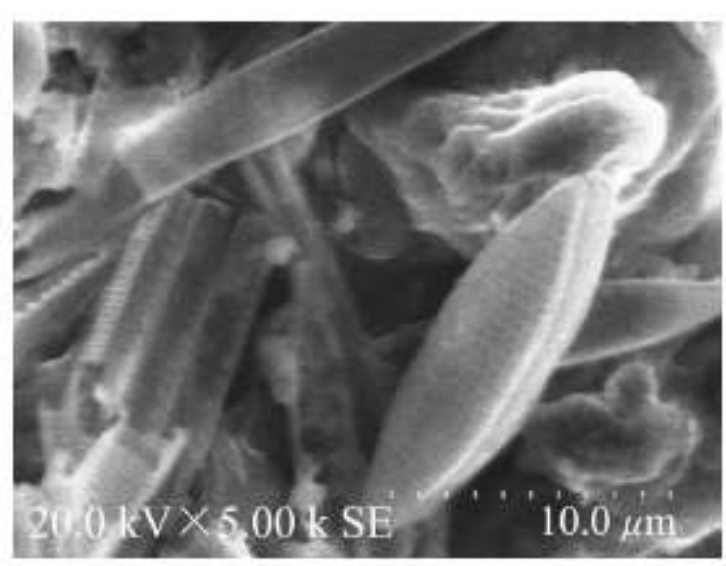

e. Sick leaf $\times 5000$

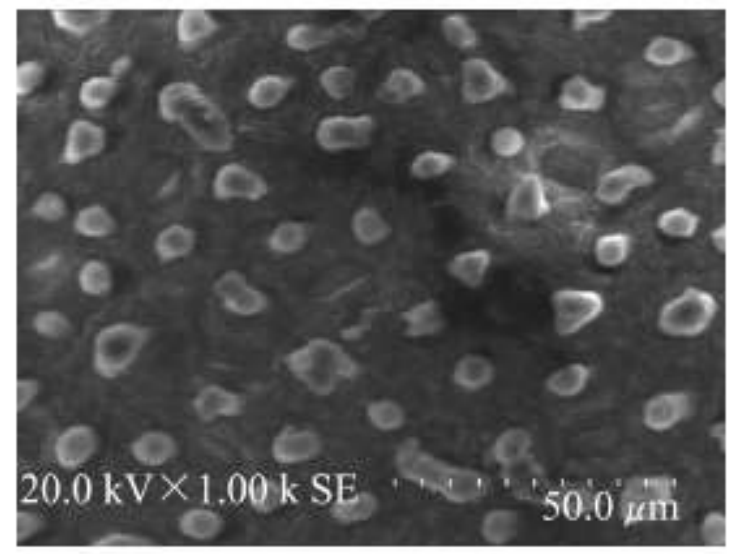

f. Dried-up leaf $\times 1000$

Fig.2 SEM of lotus leaves under different states

From the analysis of the microstructure and the surface wax fluff changes, contact angle with water change angle, the two element composite structure composed of fresh lotus leaf surface rough surface, and waxy layer, the contact angle more than 150 degrees. The performance of the superhydrophobic properties; and leaves, although the micro morphology and fresh leaves are similar, but the presence of the conical papillae, the spacing between the mastoid becomes large, although the wax content is higher than the new lotus leaf, but the leaves and the surface of the water contact angle decreases, still showed super hydrophobic properties, have further verified the root cause of the leaf wax layer is not determined lotus super hydrophobic characteristic said in the literature; the old leaves of complex surface topography no, the law of mastoid distribution, interference like battens does not cover the wax layer, causing the hydrophobic performance variation, but still has hydrophobic properties.

The lotus leaf in the old, dry and fresh leaf compared to 2 states, only mastoid and wax atrophy, not the original full, but did not change the basic structure, so the hydrophobicity change is not obvious; The 3 states are different, there is no obvious rough surface, there is almost no coverage of the wax layer, the front 3. The micro and nano structures of the four kinds of "papilla + wax layer" have changed fundamentally. According to the measurement results of contact angle, the lotus leaf has been shown to be hydrophilic".

According to the theory of Cassie, water contact lotus leaf surface, lotus leaf surface, water and surrounded by the surrounding air, forming a gas liquid solid state composite contact 3 , water at 20 DEG C of surface tension is $72 \mathrm{~m} \mathrm{~N} / \mathrm{m}$, due to significantly higher than the surface of the lotus leaf can $(30 \mathrm{~m} \mathrm{~N} / \mathrm{m})$, then the water molecules strongly in order to reduce inward contraction and air contact surface. In the micro nano composite surface structure of lotus leaf two yuan, a large number of voids and groove distribution between adjacent mastoid, the groove is filled with air, so the leaf surface structure in the formation of air layer, the organic waxy layer composed of $\mathrm{C}$ and $\mathrm{O}$ elements with low surface energy. The diameter is far greater than the micro structure of the drip of water in the leaf on the table, in the common role of wax layer and air layer under the lifting block, so that water can only form a contact and mastoid area is only $2 \% \sim 3 \%$ point contact, can not penetrate into the leaves inside, only in the leaf surface to form water beads, and reduce the friction between the two, so that the water can freely roll to form a larger contact angle and smaller roll angle, and showed the hydrophobic or super hydrophobic properties.

It is shown that the more rough the hydrophobic surface is, the less likely it is to be wetted. Therefore, the results of this study also verify this conclusion. In the fresh, old, sick, dry and other different states, the difference of the microscopic morphology and the change of the surface wax layer, determine the difference of their wettability. But from the leaf surface micro morphology and wax content contribution of 2 factors on the hydrophobic rate comparing to the same conditions and the same sample under the premise of quality, quality of lotus leaf wax old leaves, leaves and fresh lotus leaf is close, but the hydrophobicity of the two are compared with fresh leaves decreased. It is well 
known that the microscopic appearance of leaf surface is the main reason for the hydrophobicity of these 2 factors. However, the rough surface of the diseased leaves and the waxy surface of the remaining surface become the source of the wettability.

\section{Conclusion}

the surface morphology of different leaves in fresh, old, disease, dry and so on the different condition, the fresh leaves of wax layer of hair papilla structure micron uniform covered with nanometer scale, surface morphology and leaf fresh leaves are similar, the difference lies in the wax layer covering the mastoid reduced body, mastoid the hemispherical cone to change, the old leaf table are like battens of some micron in the mastoid structure, composed of rough surface on the surface of leaves, leaf surface morphology is a bit messy, micro two element composite structure of nano network structure disappeared, no villus fiber column form disease leaf surface morphology.

There are some differences in the contact angle between the lotus leaf surface in fresh, old, sick, dry and so on. The contact angle of the fresh leaves was the largest, the second of the dead leaves, and then the old leaves, and the 3 kinds of lotus leaf showed hydrophobic or super hydrophobic properties, while the diseased leaves were hydrophilic. The results showed that the difference of leaf surface wax content and leaf surface geometric morphology (roughness) was the main reason for its hydrophobic property.

There are some differences in the wax content of lotus leaf surface in different conditions, such as fresh, old, sick and dry. The wax content of old leaves and dead leaves was similar to that of fresh leaves, but the hydrophobicity was lower than that of fresh leaves. The results showed that the leaf surface microscopic morphology was the main reason for the hydrophobicity of leaf surface, and the contribution of 2 factors to the hydrophobicity of leaf surface.

\section{References}

[1] Neinhuis C, Barthlott W. Characterization and distribution of water-repellent, self-cleaning plant surfaces[J]. Annals of Botany, 1997, 79(1): 667-677.

[2] Barthlott W, Neinhuis C. The purity of sacred lotus or escape from contamination in biological surfaces[J]. Planta, 1997, 202(1): 1-3.

[3] Schulte A J, Droste D M, Koch K, et al. Hierarchically structured superhydrophobic flowers with low hysteresis of the wild pansy-new design principles for biomimetic materials[J].

Beilstein J Nanotechnology, 2011, 2(1): 228-236.

[4] Marmur A. The lotus effect: superhydrophobicity and metastability[J]. Langmuir, 2004, 20(9): $3517-3519$.

[5] Ensikat H J, Ditsche-Kuru P, Neinhuis C, et al. Superhydropho-bicity in perfection: the outstanding properties of the lotus leaf[J]. Beilstein J Nanotechnol, 2011, 2(3): 152-161.

[6] Su Y W, Ji B H, Zhang K, et al. Nano to micro structural hierarchy is crucial for state superhydrophobicand water-repellent surfaces[J]. Langmuir, 2010, 26(7): 4984-4989.

[7] Darmanin T, Guittard F. Wettability of conducting polymers: From superhydrophilicity to superoleophobicity[J]. Progress in Polymer Science, 2014, 39(1): 656-682.

[8] Favi P M, Yi S J, Lenaghan S C, et al. Inspiration from the natural world: from bio-adhesives to bio-inspired adhesives[J]. Adhesion Science and Technology, 2012,

26(8): $290-319$. 